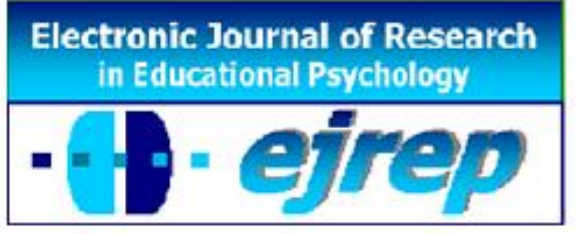

\title{
EVAPROMES, una escala para evaluar los procesos metacognitivos en escritura
}

\section{Virginia Jiménez Rodríguez ${ }^{1,2}$, María Alexandra Ulate Espinoza $^{3}$, Jesús María Alvarado Izquierdo ${ }^{1}$ y Aníbal Puente Ferreras ${ }^{1}$}

\author{
${ }^{1}$ Universidad Complutense de Madrid. \\ ${ }^{2}$ Universidad Camilo José Cela, Madrid. \\ ${ }^{3}$ Ministerio de Educación Pública de Costa Rica, San José.
}

España y Costa Rica

Correspondencia: Virginia Jiménez Rodríguez. Facultad de Trabajo Social. Campus de Somosaguas. 28224 Pozuelo de Alarcón. Madrid. España. E-mail: virginiajimenez@psi.ucm.es; María Alexandra Ulate Espinoza m.ulate.espinoza@mep.go.cr

(c) Education \& Psychology I+D+i and Ilustre Colegio Oficial de la Psicología de Andalucía Oriental (Spain) 


\section{Resumen}

Introducción. La escritura se considera hoy una herramienta básica de aprendizaje. Los procesos que subyacen a ella son complejos y la detección de la pobreza escritora de los estudiantes en las escuelas hace que se planteen nuevas formas de enseñar y aprender a escribir. Un escritor experto es consciente de cuáles son sus propios procesos de aprendizaje, cómo funcionan, y conoce cómo optimizar su funcionamiento y el control de sus propios procesos escritores.

Método. La prueba EVAPROMES incluye 28 ítems que representan "situaciones de escritura" con tres alternativas de respuesta que se valoran ante las que el escritor debe tomar partido. La alternativa seleccionada permite conocer "cómo los escritores se perciben" y "cómo creen que actuarían" ante posibles dilemas que se les plantean a la hora de escribir, así como su grado de motivación ante el acto escritor. Cada alternativa expresa un grado diferente de conciencia escritora. La prueba ha sido validada en muestra costarricense y española, compuesta por 1442 estudiantes entre 10 y 15 años (915 costarricenses y 527 españoles).

Resultados. Los resultados indican que EVAPROMES es un instrumento que permite recoger información del grado o nivel de conciencia escritora en niños y adolescentes, con una medida precisa de los procesos implícitos, permitiendo un buen pronóstico del rendimento en escritura y académico en general.

Conclusión. EVAPROMES permite identificar al alumnado con baja y alta conciencia escritora. Basándose en esta identificación, se pueden diseñar programas de intervención específicos en estrategias metacognitivas en el área de la escritura adaptando la respuesta educativa a los alumnos evaluados.

Palabras Clave: Procesos Metacognitivos, Escritura, Planificación, Autorregulación, Evaluación, Estrategias. 


\title{
EVAPROMES, an assessment scale for metacognitive processes in writing
}

\begin{abstract}
Introduction. Writing is considered a basic tool of learning today. The processes that underlay to it are complex and detecting the writer poverty of students in schools makes new ways of teaching and learning to write arise. A skilled writer is aware of what their own learning processes are, how they work, and how to optimize the performance and control of their own writers processes.
\end{abstract}

Method. EVAPROMES test includes 28 items that represent "writing situations" with three possible answers in which the writers must select one of them. The alternative selected allows to know "the way the writers perceive themselves" and "how the writers believe that they would act" against possible dilemmas they face when writing as well as their level of motivation when they write. Each alternative expresses a different level of writer awareness. The test has been validated with Costa Rican and Spanish samples, the total sample was 1442 students aged 10 to 15 years old (915 Costa Ricans and 527 Spanish).

Results. The results show EVAPROMES is a tool that allows you to collect information about student's level in children and adolescents of writing awareness, with an accurate measure of implicit processes, allowing a good prediction the writing and academic performance in general.

Conclusion. EVAPROMES identifies students with low and high writer awareness. Based on this identification you can design specific intervention programs in metacognitive strategies in the area of writing adapting the educational answer to students evaluated.

Keywords: Metacognition Processes, Writing, Planning, Self-Regulation, Assesment, Strategies.

Reception: 08.09.15 Initial acceptance: 09.16.15 Final acceptance: 11.19.15 


\section{Introducción}

De las actividades cognitivas específicas del ser humano, la escritura es sin duda una de las más complejas. Esta complejidad de la tarea es una de las causas que explica por qué resulta tan difícil utilizar la escritura a la hora de expresar ideas, sentimientos o argumentos (Graham y Harris, 1996). La creatividad y la productividad como elementos esenciales de la escritura se encuentran muy menguadas en la escuela y en la vida. Aunque los alumnos y los maestros reconocen la importancia de la escritura como actividad para mejorar el pensamiento, la inteligencia humana y el éxito escolar, también reconocen que sus habilidades como escritores son muy pobres (Shell, Murphy y Bruning, 1989).

Según Mata (1997), existen tres temas que estructuran el campo de la escritura: 1) la evaluación de las composiciones (el más antiguo y quizás el más desarrollado); 2) el análisis de los procesos psicológicos implicados en la composición; y 3) la evaluación de las estrategias didácticas para enseñar la composición. Estos temas se corresponden, de alguna manera, con tres enfoques de la enseñanza: la escritura como producto; la escritura como proceso; y la escritura inmersa en el contexto. Cada uno de estos enfoques, desde su peculiar visión, aporta datos y respuestas sobre el qué, cómo, y cuándo enseñar y aprender el proceso de la escritura.

La escritura como proceso ha sido explicada por modelos cognitivos contemporáneos (Flower y Hayes, 1980; Scardamalia y Bereiter, 1992). Estos modelos describen las operaciones mentales que ocurren cuando se escribe. En este sentido, estudian tres subprocesos (planteamiento, redacción y revisión), las diferencias entre escritores expertos y principiantes y las estrategias de aprendizaje y enseñanza. La tarea de redactar un texto coherente y adecuado implica varias y recurrentes etapas en las que el que escribe debe coordinar un conjunto de procedimientos específicos: 1) Planteamiento (propósito del escrito, previsible lector, contenido); 2) Redacción o textualización (características del tipo de texto, léxico adecuado, morfosintaxis normativa, cohesión, ortografía, signos de puntuación); y 3) Revisión (el volver sobre lo ya escrito, releyendo y evaluándolo). El modelo de Linda Flower y John Hayes (1980), resulta muy útil para describir cómo se relacionan el pensamiento y el lenguaje en la escritura. Este modelo define la escritura como una actividad de solución de problemas en la que intervienen la interacción de tres elementos: el entorno de la tarea, la 
memoria a largo plazo y la memoria de trabajo. Para realizar las operaciones comprendidas en la escritura, la persona necesita utilizar tanto la memoria de trabajo como la de largo plazo. Deberá utilizar la de trabajo de la manera que plantea Hayes (1996), siendo consciente de que el escritor necesita almacenar la información que resulta de la ejecución de las etapas de elaboración, expresión y revisión, así como también utilizar esa información para conducir las operaciones mentales comprendidas en cada una de esas etapas. Esto significa que, a medida que escribe, el escritor deberá utilizar permanentemente información proveniente de la memoria de trabajo. De igual manera, el escritor, a medida que ejecuta las diferentes etapas del proceso de la escritura, necesita utilizar información almacenada en su memoria a largo plazo: evocar información para determinar el contenido a incluir en el texto durante la elaboración, utilizar los códigos, diferentes fuentes, recursos y competencias linguiísticas durante la expresión o conducir las diversas acciones que tienen lugar durante la revisión.

La toma de conciencia de las fases de desarrollo de la escritura constituye los elementos que sustenta la teoría moderna de la escritura eficaz (metaescritura). Una persona utilizará su conciencia para escribir cuando lleva a cabo este proceso, primero, se mantenga consciente de las etapas que él mismo comprende $\mathrm{y}$, segundo, se mantenga alerta para supervisar, evaluar y regular la ejecución de su escritura.

\section{Etapas de la escritura}

Conocer las etapas de la escritura es fundamental para desarrollar la metaescritura. Son las siguientes: planteamiento (o elaboración), redacción (o expresión) y revisión:

1) El planteamiento es una actividad de pre-escritura o elaboración, durante la cual el individuo concibe o genera las ideas que conformarán el texto que desea escribir y, luego, lo representa en su mente. Es en esta etapa cuando el escritor establece la direccionalidad de lo que va a escribir.

2) La redacción es la etapa en la cual quien escribe se vale de sus habilidades para producir la versión escrita del texto; es decir, traduce el texto que tiene representado en su mente a palabras y éstas a su expresión gráfica (Galbraith y Torrance, 1999; Graham y Harris 2000). Como una posición general sobre lo que acontece en esta etapa, conviene tener claro que un texto per se no tiene significado alguno. Un texto es, en su inicio, un conjunto de 
pistas (códigos) de variada naturaleza que guían al lector para que éste construya su propio significado.

3) En la última etapa de revisión, el escritor se aboca a leer repetidamente lo escrito, manteniendo una actitud crítica, para luego ajustar las partes que le parecen insatisfactorias. La revisión permite al autor del texto utilizar su propio criterio para asegurarse, en primer lugar de que hay congruencia entre lo expresado en el texto y la direccionalidad que se le quería dar a éste. En segundo lugar, asegurarse de que existe coherencia entre las diferentes partes del texto, así como entre lo expresado en éste y su manera de ser y de pensar. En tercer lugar, asegurarse de que la información presentada es suficiente en cantidad y, a su juicio, que en ella no hay excesos ni carencias; que lo escrito es adecuado y comprensible para la mayoría del público al cual está siendo dirigido y que su redacción es suficientemente fluida, armónica y elegante. Finalmente, el escritor debe asegurarse de que el texto posee las características aceptables en cuanto al lenguaje utilizado; es decir, si los elementos del lenguaje son empleados de manera apropiada.

\section{Estrategias metacognitivas en escritura}

Pero para elaborar un texto escrito coherente y comprensible no es suficiente con conocer las etapas por las que se va pasando al escribir. Es necesario también ser consciente de que cuanto mayor sea el conocimiento de lo que sabemos, del porqué de nuestros aciertos o desaciertos, mayores serán las posibilidades de aprender, consolidar éxitos, superar deficiencias y disminuir la dependencia del azar o suerte. Esto es lo que desemboca en la metaescritura: ser consciente de qué se escribe y cómo se escribe, así como remediar fallas utilizando estrategias metacognitivas. A raíz de los argumentos propuestos por Graves (1996) y Heller y Thorogood (1995) se establece una clasificación de estrategias metacognitivas de producción de textos o captación consciente de las capacidades y limitaciones de los procesos de pensamiento que originan un texto escrito. Las estrategias metacognitivas sugeridas son:

1) Antes de escribir:

- Explicar el objetivo que se tiene cuando se escribe.

- Construir ideas acerca del contenido del texto y su relación con los conocimientos previos.

- Tomar conciencia sobre: ¿para quién estoy escribiendo?, ¿qué tipo de texto quiero escribir?, ¿por qué escribo? 
2) Durante la escritura:

- Identificar las dificultades que impiden expresar las ideas.

- Utilizar palabras, oraciones y párrafos para comunicarse.

- Asegurar la coherencia y cohesión del texto.

- Seleccionar ideas principales y secundarias.

\section{3) Después de la escritura:}

- Analizar la calidad del contenido: amplitud, riqueza, profundidad y precisión de las ideas.

- Revisar la organización y presentación del contenido: estructura del texto y de las unidades que lo componen.

- Confirmar o rechazar el estilo de la escritura: adecuación a la audiencia y a la finalidad.

- Corregir aspectos morfológicos, sintácticos, léxicos y ortográficos.

El comprender y construir una composición escrita son tareas que exigen un pensamiento estratégico por parte del estudiante; es decir, el escritor debe tener capacidad para dominar técnicas y además saber modificarlas para adaptarlas a situaciones cambiantes (Solé y Teberosky, 2010). Este pensamiento estratégico incluye funciones ejecutivas como la capacidad de inhibir respuestas automáticas, la solución de problemas, la planificación de tareas, el desarrollo e implementación de estrategias y el saber alternarlas cuando la ocasión lo requiere (flexibilidad cognitiva), y la memoria de trabajo.

Las funciones ejecutivas se pueden definir como el conjunto de habilidades cognitivas que permiten la anticipación y el establecimiento de metas, el diseño de planes y programas, el inicio de las actividades y de las operaciones mentales, la autorregulación y monitoreo de las tareas, la flexibilidad en el trabajo cognitivo y su organización en el tiempo y el espacio. Todo ello para llevar a cabo una conducta eficaz, creativa y aceptada socialmente (TirapuUstárroz y Muñoz-Céspedes, 2005). Las habilidades metacognitivas describen principalmente procesos de control ejecutivo. Son procedimientos que permiten a las personas encadenar de forma eficaz las acciones que les llevan a realizar con éxito una tarea. Incluyen habilidades de regulación de los procesos cognitivos, entre los que cabe distinguir la planificación, la supervisión y la evaluación (Flavell y Wellman, 1977). 
Finalmente, las funciones ejecutivas metacognitivas son el conjunto de procesos neurobiológicos y cognitivos que dan origen a la capacidad mental que permite la elaboración interna de control ejecutivo que fundamenta los procesos de autorreflexión y autoconciencia para elaborar la respuesta final que, de forma eficaz, resuelva el problema. La metacognición también se ha abordado desde tres tipos de conocimiento: el conocimiento declarativo (qué conocimientos o acciones se deben llevar a cabo para realizar una tarea); el conocimiento procedimental (cómo se pueden llevar a cabo esas acciones); y el conocimiento condicional (se refiere al cuándo y al porqué utilizar una estrategia y no otra para la ejecución de una determinada tarea).

\section{Funcionamiento metacognitivo de la escritura}

Según Karmiloff-Smith (1995) una constante redescripción representacional de los conocimientos a lo largo del desarrollo, da origen al modelo de cambio evolutivo basado en la idea de que cada vez el conocimiento será más explícito y accesible, siendo esas representaciones en un principio de carácter procedimental hasta que el niño logra dominarlas para explicarlas y transformarse en representaciones declarativas, tomando este postulado gran importancia en el área de la metacognición; es decir, múltiples recodificaciones del conocimiento en la memoria a largo plazo permiten alcanzar niveles más altos de conciencia metalingüística.

Basándose en la teoría de Karmiloff-Smith (1995), las investigadoras Flórez, Mondragón, Pérez y Torrado (2003) plantean tres niveles de funcionamiento metacognitivo en la escritura, donde desglosan las características de los tres procesos metacognitivos (planteamiento o planificación, autorregulación o supervisión y evaluación) en función de cada nivel, considerando también los conocimientos que tiene el escritor sobre sus propios recursos cognitivos a la hora de abordar una tarea de producción escrita. La autorregulación o supervisión se identifica con el proceso escritor de redacción y la evaluación con el de revisión.

El funcionamiento metacognitivo del primer nivel o nivel I en escritura es de tipo procedimental donde el tipo de redacción del escritor a nivel explícito es todavía burdo con pérdida de numerosos detalles de la información manejada en sus descripciones. El manejo de los conocimientos se produce a nivel implícito (Karmiloff-Smith, 1995). En este nivel, la planificación se caracteriza por el planteamiento de, al menos, un objetivo específico y de las estrategias conocidas para llevar a cabo con éxito la tarea. El proceso de autorregulación per- 
mite mantener en la conciencia el objetivo planteado anteriormente, corrigiendo, si fuera necesario, el contenido para una mejor comprensión del mismo. Es en este proceso cuando se produce la escritura propiamente dicha. Finalmente, el escritor revisa (evalúa) el contenido de lo escrito pensando en sus posibles lectores.

El funcionamiento metacognitivo del segundo nivel o nivel II en escritura se caracteriza por la posibilidad de llevar a la conciencia los conocimientos ya adquiridos, pero no siempre se reflexiona sobre ellos de una forma consciente, ni se verbalizan. Es un nivel intermedio. En este nivel, el proceso de planificación lleva implícito el cuestionamiento acerca de las características que debe tener el texto y lo que se pretende conseguir con la redacción. A continuación se evocan los conocimientos necesarios y se pone en marcha, durante la escritura, alguna estrategia para desarrollar la tarea con éxito, corrigiendo, si fuera necesario, la forma del escrito para una mejor comprensión del mismo; finalmente se identifican y evalúan las características generales del texto que cumplen con el objetivo planteado con anterioridad.

El funcionamiento metacognitivo del tercer nivel o nivel III en escritura es el más complejo. Los conocimientos adquiridos sobre escritura están en la memoria a largo plazo por lo que se pueden llevar a la conciencia y manejarlos con el fin de reflexionar sobre ellos, analizarlos y expresarlos verbalmente. Para planificar, el escritor se cuestiona acerca de las características del texto así como los conocimientos que posee del tema. El proceso de autorregulación hace que el escritor implemente una estrategia general y al menos dos estrategias más específicas. Mientras escribe, utiliza sus conocimientos previos ya evocados y simultanea la identificación y la corrección de los contenidos escritos con el fin de hacer más comprensible su texto. Una vez terminada la tarea, se realiza una revisión o evaluación de lo escrito donde se producen cambios de, al menos, uno de los contenidos del texto para facilitar la comprensión de la audiencia.

\section{Objetivo}

El presente estudio pretende examinar las características de EVAPROMES (UlateEspinoza, Jiménez-Rodríguez, Alvarado-Izquierdo y Puente-Ferreras, 2015) una prueba destinada a evaluar la percepción de los escritores y sus capacidades escritoras así como identificar a escolares con baja y alta conciencia escritora entre alumnado de España y Costa Rica. La prueba permite evaluar los procesos que se ponen en marcha ante una tarea de escritura creativa en detrimento de la escritura mecánica, centrándose en la metaescritura 
como motor de cambio y mejora de la calidad de la producción escrita. Los elementos de diagnóstico extraídos de la prueba permiten definir las líneas de actuación para desarrollar programas de aprendizaje e intervención en escritura.

\section{Método}

\section{Participantes}

Participaron en el estudio 1442 estudiantes, 915 costarricenses y 527 españoles de edades comprendidas entre los 10 y los 15 años. La muestra de Costa Rica fue una muestra representativa de los estudiantes que cursan sexto grado en el país ya que se siguió un procedimiento de muestreo aleatorio sistemático en el que participaron 31 centros educativos seleccionados del total de 155 escuelas que conforman el Departamento Pedagógico de la Dirección Regional de Educación de Occidente (DREO).

En España, participaron centros públicos, concertados y privados lo que permite disponer de datos acordes a la diversidad y realidad de la escolarización en la Comunidad Madrid. (56\% públicos, $24 \%$ concertados y $20 \%$ privados). La muestra fue de 527 estudiantes (51.9\% varones, $48.1 \%$ mujeres), el 60\% de ellos cursaban $6^{\circ}$ de Educación Primaria y el resto Educación Secundaria Obligatoria (ESO), la selección se realizó procurando que los rangos de edad de los integrantes de la muestra española fueran similares a la de los integrantes de la muestra costarricense, con edades comprendidas entre los 10 y los 15 años, con una edad media de 11.71 años $(\mathrm{Dt}=1.17)$. Nótese que sexto grado en Costa Rica coincide, dependiendo de las zonas, con las edades en la que se cursa en España sexto de primaria o primero de secundaria.

\section{Instrumentos}

Composición o calidad de la producción escrita. Se elaboró una prueba que consistía en la redacción libre de un cuento o historia fantástica. Para evaluar la calidad de los escritos se empleó un sistema de jueces que debían valorar 13 criterios de calidad de los escritos en un rango de 0 a 2 puntos. El procedimiento fue probado y refinado en sucesivas aplicaciones hasta que se observó un acuerdo prácticamente unánime entre los jueces al valorar los escritos de prueba. 
La escala de conciencia lectora (ESCOLA; Puente-Ferreras, Jiménez-Rodríguez y Alvarado-Izquierdo, 2009). Para evaluar la relación con otras variables metacognitivas y disponer de evidencia de validez convergente se utilizó ESCOLA, prueba que está indicada para evaluar el nivel de metacognición en lectura en escolares (ver Jiménez-Rodríguez, Puente- Ferreras, Alvarado- Izquierdo y Arrebillaga-Durante, 2009).

Clima motivacional en el aula. Para la medida del clima motivacional se utilizó el cuestionario de clima motivacional de clase (CMC-Q) desarrollada por Alonso-Tapia y Fernández (2008). La investigación de los autores sobre estructura de metas de clase ha mostrado la necesidad de evaluar el clima motivacional para facilitar la valoración de las actividades educativas y promover su cambio. Las características psicométricas del CMC-Q son satisfactorias, habiéndose obtenido altos valores de consistencia interna en diferentes muestras de entre .92 y .93 .

Rendimiento académico. Se tomaron los promedios anuales de los escolares en diversas materias: sociales, ciencias, matemáticas, inglés y castellano.

\section{Procedimiento}

Con el objetivo de examinar los fundamentos teóricos, las fortalezas y limitaciones prácticas, conceptuales y psicométricas de EVAPROMES se realizaron varias pruebas en Costa Rica, específicamente en las Direcciones Regionales de Enseñanza de Alajuela (DREA) y de Occidente (DREO) y en España en la Comunidad de Madrid. A continuación se sintetiza el proceso realizado para la validación de la escala EVAPROMES, el cual se organizó en dos etapas:

Primera etapa (versión piloto). La primera versión de EVAPROMES contó con un diseño de escala de autoreporte compuesta por 50 ítems, los cuales fueron elegidos en función de su relevancia y representatividad en relación a los tres procesos metacognitivos descritos previamente y la experiencia práctica expresada por docentes e investigadores en entrevistas y grupos focales. Además, la revisión teórica y gramatical de esta escala contó con el aporte y revisión de investigadores expertos de la Universidad Complutense de Madrid (UCM), la Universidad Autónoma de Madrid (UAM) y la Universidad de Barcelona (UB), con quienes se mantuvo una discusión académica sobre cada reactivo con el fin de mejorar la escala. 
El diseño de respuesta estuvo constituido por una escala tipo Likert de cinco puntos. Esta primera versión piloto se aplicó en una muestra elegida de forma intencionada, la cual estuvo conformada por 210 estudiantes costarricenses y 260 estudiantes españoles que cursaban quinto y sexto de Educación Primaria. La muestra total estuvo representada por un 63\% de hombres y $37 \%$ de mujeres de edades comprendidas entre los 10 y 15 años. Los análisis factoriales exploratorios apuntaban a una estructura unidimensional con una alta fiabilidad (alfa de Cronbach de 0.92) que no permite diferenciar adecuadamente entre los tres procesos lectores. Además, se observaron indicios de sesgo de respuesta por deseabilidad social lo que se atribuyó al tipo de escala de respuesta (tipo Likert) que se había utilizado, ya que este tipo de escala facilita asociar las puntuaciones altas (en ítems directos) o bajas (en ítem inversos) como alternativas más deseables.

Segunda etapa (versión definitiva). Se tomaron los 28 ítems que mejor funcionamiento habían mostrado en la etapa 1 , si bien ahora la respuesta a cada reactivo debía hacerse en una escala de elección múltiple en donde cada una de las opciones (a, b, c) representaba diferentes estrategias que revelaban un nivel diferente de conciencia metacognitiva. El instrumento en su versión final se administró, junto a los instrumentos anteriormente mecionados, a muestras amplias y representativas tanto en Costa Rica como en España, observándose que el nuevo instrumento presentaba una mejor diferenciación entre los procesos de lectura, estaba libre del sesgo de deseabilidad social y observándose adecuadas evidencias de validez y una aceptable fiabilidad como se muestra en el apartado de resultados.

\section{Análisis de datos}

Se realizaron análisis de validez y fiabilidad de las puntuaciones obtenidas en ambos países. Respecto a la validez se utilizaron técnicas de análisis factorial confirmatorio (AFC) y AFC multigrupo para establecer la invarianza de la medida entre países, y entre otras variables relevantes como sexo y edad. También se recogieron evidencias de validez convergente con medidas de constructos relacionados como son ESCOLA y la escala de motivación CMCQ. Finalmente, la fiabilidad por consistencia interna se estimó mediante el procedimiento estándar del coeficiente $\alpha$ de Cronbach y los procedimientos omega de McDonnald y GLB (gran límite inferior de la fiabilidad) implementados en el programa Factor 9.3 (Lorenzo-Seva y Ferrando, 2006). 


\section{Resultados}

\section{Análisis estructural y validez de constructor}

La fundamentación teórica de EVAPROMES nos indica una medida compleja ya que en su diseño se elaboraron ítems correspondientes a los procesos metacognitivos de Planificación, Supervisión y Evaluación aplicados a las variables Texto, Tarea y Persona. A pesar de la complejidad inherente al instrumento los análisis de la primera etapa mostraban una alta fiabilidad, que como veremos en el apartado fiabilidad se confirman en los análisis de esta segunda etapa, acorde al objetivo de diseñar elementos que permitan medir un constructo global que denominaremos Las estrategias metacognitivas aplicadas a la escritura, identificándose tres procesos subyacentes relacionados entre sí (planificación, supervisión y evaluación).

EVAPROMES está compuesto por 28 ítems que se responden en una escala ordinal. Los ítems se elaboraron siguiendo un diseño de 3 procesos x 3 variables que permite obtener nueve agrupaciones o parcelas de ítems: (1) PLATEX ítems que miden planificación aplicado a la variable texto, (2) PLATAR ítems que miden planificación aplicado a la variable tarea, (3) PLAPER ítems que miden planificación aplicado a la variable persona, (4) SUPTEX ítems que miden supervisión aplicado a la variable texto, (5) SUPTAR ítems que miden supervisión aplicado a la variable tarea, (6) SUPPER ítems que miden supervisión aplicado a la variable persona, (7) EVATEX ítems que miden evaluación aplicado a la variable texto, (8) EVATAR ítems que miden evaluación aplicado a la variable tarea y (9) EVAPER ítems que miden evaluación aplicado a la variable persona.

Mediante AFC y aplicando máxima verosimilitud robusta se evaluó la bondad de ajuste tanto al modelo unidimensional como al de tres factores (Planificación, Supervisión y Evaluación).

Tabla 1. Bondad de ajuste de los modelos propuestos para EVAPROMES

\begin{tabular}{lcccccc}
\hline Modelo & $\chi^{2}(\mathrm{gl})$ & Probab. & RMSEA & SRMR & NNFI & CFI \\
\hline Un único factor & $71.73(27)$ & $<.01$ & .04 & .04 & .94 & .95 \\
$\begin{array}{l}\text { Tres factores correla- } \\
\text { cionados }\end{array}$ & $57.48(24)$ & $<.01$ & .04 & .03 & .95 & .96 \\
$\begin{array}{l}\text { Tres factores correla- } \\
\text { cionados con errores } \\
\text { correlacionados }\end{array}$ & $28.29(21)$ & .13 & .02 & .02 & .99 & .99 \\
\hline
\end{tabular}

Nota: RMSA = Root Mean Square Error of Approximation, SRMR= Root Mean Square Residual; NNFI= NonNormed Fit Index; CFI = Comparative Fit Index. 
En la Tabla 1 podemos ver que si bien el ajuste a un único factor (modelo unidimensional) es aceptable (valores RMSEA y SRMR menores de .08 y valores de NNFI y CFI a partir de .95), la estructura tridimensional supone una mejora estadísticamente significativa en términos de $\chi^{2}=14.25, p<.01$. Adicionalmente, se obtiene una mejora sustancial, en términos de ajuste de $\chi^{2}$ al introducir correlaciones entre errores de las variables Texto y Tarea (ver Figura 1).

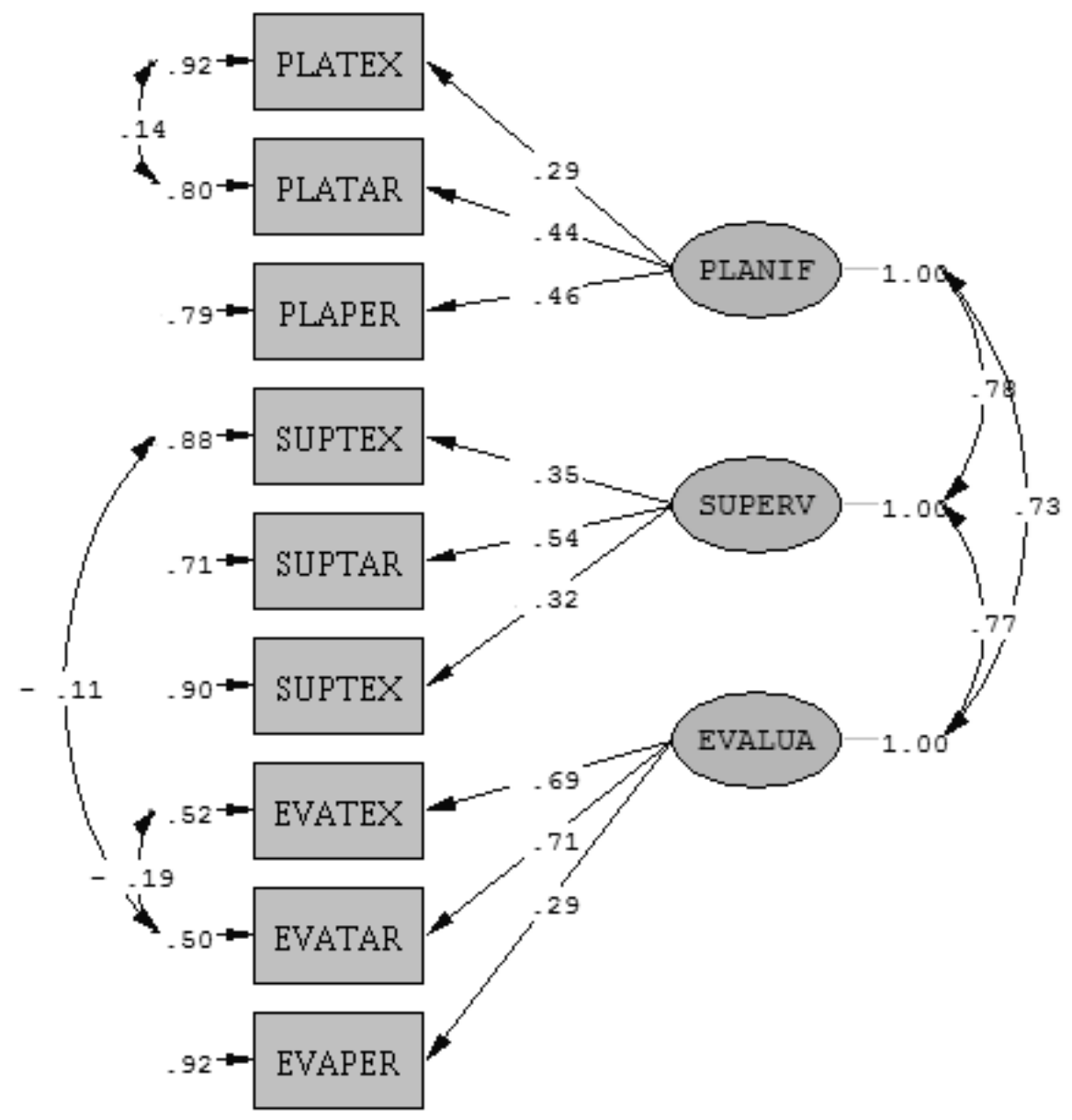

Figura 1. Modelo de tres factores para EVAPROMES

En la Figura 1 se visualiza el modelo que mejor ajuste presenta tanto a nivel estadístico como sustantitvo. Por una parte, la correlación media entre factores de .75 es adecuada para a la vez considerar que hay una importante varianza común, la medida de metacognición aplicada a la lectura, y a la vez poder discernir entre procesos que forman parte de esta medida. La existencia de solapamientos (correlación entre errores) entre las variables contextuales de Tarea y Texto se explica en que ambas comparten elementos comunes que son fácilmente diferenciables de la variable o aspectos relativos a la persona. 
El modelo representado en la Figura 1 se obtuvo con los datos de la muestra costarricense que era la más amplia, si bien la bondad de ajuste a este modelo también se observó en la muestra española $\chi^{2}(3)=13.70 p=.88$, RMSEA $<.01$. No obstante, para una evaluación más sistemática de la invarianza, a continuación se muestran los resultados de los AFC multigrupo en los que se puso a prueba la equivalencia métrica del test en los distintos grupos y poblaciones estudiadas.

\section{Estudios de equivalencia métrica e invarianza de la medida}

En los estándares para la elaboración de test psicológicos y educativos publicados por las tres grandes asociaciones americanas (APA, AERA, NCME, 2014) se considera un aspecto clave para la validación de las pruebas el poder mostrar que estas cumplen con el requisito de equidad ofairness. Es preciso evitar sesgos en la interpretación de las puntuaciones cuando se comparan diversos grupos, no siendo admisible si antes no se ha mostrado que se está midiendo el mismo constructo, esto es, que hay equivalencia métrica o invarianza de la medida. Existen distintos niveles de invarianza, el más básico es la invarianza de configuración que consiste en verificar que en los grupos el mismo modelo ajusta a los datos, un nivel de invarianza más exigente es la métrica, cuando los pesos de los ítems y sus fiabilidades son similares en los grupos, cuando además coinciden las medias se habla de invarianza métrica y el nivel superior de invarianza es la escalar cuando incluso las varianzas error son iguales entre grupos.

\section{Estudio de Invarianza entre la muestra española y costarricense}

En la Tabla 2 se indican los índices de bondad de ajuste de los AFC multigrupo con estimación por máxima verosimilitud robusta.

Tabla 2. AFC multigrupo muestra española y costarricense.

\begin{tabular}{lcccccc} 
INVARIANZA & $\chi^{2}(\mathrm{gl})$ & $p$ & RMSEA & SRMR & NNFI & CFI \\
\hline Configuración & $53.36(45)$ & .18 & .02 & .02 & .99 & .99 \\
Métrica & $73.80(51)$ & .02 & .03 & .04 & .98 & .99 \\
Métrica y Covarianzas & $84.10(54)$ & .01 & .03 & .05 & .98 & .98 \\
Escalar & $266.35(57)$ & $<.01$ & .07 & .04 & .85 & .88 \\
\hline
\end{tabular}

El modelo de invarianza métrica con iguales covarianza muestra un buen ajuste como se puede apreciar en la Tabla 2, si bien su bondad de ajuste es ligeramente inferior al modelo 
de configuración y estadísticamente mejor que el modelo escalar. En consecuencia, se puede afirmar que la medida del constructo metacognición en escritura es invariante para las dos muestras analizadas a nivel de configuración y métrico siendo legítimo hacer comparaciones entre los grupos con respecto al nivel de conciencia en metacognición.

Tanto en la muestra española como en la costarricense se observó que entre los diferentes colegios existen grandes diferencias en el nivel promedio de conciencia en escritura y que estas diferencias se relacionaban con la preocupación en los centros por la didáctica en estrategias metacognitivas. Observamos, a su vez, que los centros mejor valorados por sus resultados académicos en Costa Rica y en España (pruebas CDI de sexto de Educación Primaria) eran los que obtenían las puntuaciones medias más elevadas en EVAPROMES.

\section{Estudio de Invarianza entre sexos}

En la Tabla 3 se indican los índices de bondad de ajuste para los distintos modelos de invarianza analizados.

Tabla 3. AFC multigrupo: hombres vs mujeres.

\begin{tabular}{lcccccc}
\hline INVARIANZA & $\chi^{2}(\mathrm{gl})$ & $p$ & RMSEA & SRMR & NNFI & CFI \\
\hline Muestra española & & & & & & \\
Configuración & $50.54(45)$ & .26 & .02 & .03 & .99 & .99 \\
Métrica & $60.39(51)$ & .17 & .03 & .05 & .98 & .99 \\
Métrica y Covarianzas & $63.13(54)$ & .19 & .03 & .05 & .98 & .99 \\
Escalar & $113.54(57)$ & $<.01$ & .06 & .04 & .90 & .92 \\
& & & & & & \\
Muestra costarricense & & & & & & \\
Configuración & $49.83(45)$ & .29 & .02 & .03 & .99 & .99 \\
Métrica & $59.21(51)$ & .20 & .02 & .04 & .98 & .99 \\
Métrica y Covarianzas & $62.92(54)$ & .19 & .02 & .04 & .98 & .99 \\
Escalar & $84.54(57)$ & .01 & .03 & .03 & .95 & .96 \\
\hline
\end{tabular}

En la Tabla 3 podemos observar un buen ajuste tanto en el modelo de configuración como en el de invarianza métrica, e incluso fijando las covarianzas entre los tres procesos. Solo se observan diferencias estadísticamente significativas al comparar los modelos anteriores con el de invarianza escalar (este modelo ya no muestra bondad de ajuste en chi-cuadrado 
con $p<.05$ ni tampoco en los valores de NNFI $<.95$ y CFI $<.95$ en la muestra española). Que no exista invarianza escalar era lo esperado ya que las mujeres en las pruebas verbales suelen obtener puntuaciones superiores a los hombres y en el caso de EVAPROMES también ocurre.

Estudio de Invarianza entre Educación Primaria y Educación Secundaria Obligatoria. En la muestra española además de estudiantes de sexto de Educación Primaria, se administró el test a estudiantes del primer ciclo de Educación Secundaria Obligatoria (primero y segundo de ESO) lo que nos permite hacer una evaluación de la invarianza entre estas dos etapas educativas. (Ver Tabla 4).

Tabla 4. AFC multigrupo muestra española: hombres vs mujeres.

\begin{tabular}{lcccccc}
\hline INVARIANZA & $\chi^{2}(\mathrm{gl})$ & $p$ & RMSEA & SRMR & NNFI & CFI \\
\hline Configuración & $46.34(45)$ & .42 & .01 & .04 & 1.00 & 1.00 \\
Métrica & $44.58(48)$ & .61 & $<.01$ & .04 & 1.00 & 1.00 \\
Métrica y Covarianzas & $49.35(51)$ & .54 & $<.01$ & .06 & 1.00 & 1.00 \\
Escalar & $61.95(57)$ & .30 & .02 & .07 & .99 & .99 \\
\hline
\end{tabular}

En la comparación de los estudiantes de sexto de Educación Primaria con los de Educación Secundaria Obligatoria se obtiene invarianza escalar lo que justifica aplicar el instrumento en el rango de edades que va desde los 10 a los 15 años de edad.

Fuentes de evidencia externas: validez predictiva y relación con otras variables

En este apartado se muestran las evidencias externas sobre la utilidad de EVAPROMES en la predicción de la calidad de los escritos producidos por los escolares y su rendimiento académico. A su vez se mostrará la relación de EVAPROMES con otras medidas de metacognición y otras variables relacionadas como el clima motivacional. Mediante análisis de los patrones de correlación y de regresión mostramos a continuación la relación de EVAPROMES con las siguientes medidas e instrumentos: composición o calidad de la producción escrita, ESCOLA, Clima motivacional y rendimiento académico. Para este estudio se tomó una submuestra de 349 escolares de sexto curso a los que se aplicaron las pruebas que se indican en la Tabla 5. 
Tabla 5. Patrones de correlación entre las pruebas de metacognición y los criterios Composición y Rendimiento académico.

\begin{tabular}{|c|c|c|c|c|c|c|c|c|}
\hline & ESCOLA & Clima & $\begin{array}{l}\text { Composi- } \\
\text { ción }\end{array}$ & $\begin{array}{l}\text { Ciencias } \\
\text { Sociales }\end{array}$ & $\begin{array}{l}\text { Ciencias } \\
\text { Naturales }\end{array}$ & $\begin{array}{l}\text { Matemá- } \\
\text { ticas }\end{array}$ & $\begin{array}{l}\text { Lengua } \\
\text { Inglesa }\end{array}$ & $\begin{array}{c}\text { Lengua } \\
\text { Castellana }\end{array}$ \\
\hline EVAPROMES & $.42 * * *$ & $.22 * * *$ & $.64 * * *$ & $.31 * * *$ & $.33 * * *$ & $.34 * * *$ & $.28 * * *$ & $.37 * * *$ \\
\hline ESCOLA & - & $.25 * * *$ & $.41 * * *$ & $.31 * * *$ & $.36 * * *$ & $.29 * * *$ & $.31 * * *$ & $.34 * * *$ \\
\hline CLIMA & & - & $.16^{* *}$ & $.16^{* *}$ & .11 & $.14^{*}$ & .10 & $.19 * *$ \\
\hline
\end{tabular}

En la Tabla 5 se observa que existe una correlación de tamaño medio entre EVAPROMES y ESCOLA, mientras que con clima motivacional la relación aunque significativa ( $p<.001)$ es mucho más débil como era de esperar. La correlación entre EVAPROMES y ESCOLA de .42 nos indica que hay una parte de varianza común entre ambas medidas (un $18 \%$ aproximadamente), en coherencia con el propósito de evaluar diferentes expresiones de las habilidades metacognitivas, en el caso de ESCOLA la conciencia lectora y en el de EVAPROMES la escritora.

En cuanto a la validez predictiva de EVAPROMES, observamos que alcanza una correlación con la medida de composición (calidad de la producción escrita) de .64 con lo que se evidencia su utilidad para pronosticar este criterio. Para conocer los procesos de EVAPROMES que tienen mayor valor predictivo sobre la medida de composición se realizó un análisis de regresión (ver Tabla 6).

Tabla 6. Modelo de regresión para las tres medidas de EVAPROMES.

\begin{tabular}{lcccc}
\hline & \multicolumn{2}{c}{ Coeficientes no } & \multicolumn{2}{c}{ Coeficientes } \\
tipificados & \\
& $B$ & Estandarizados & $\beta$ & $t$ \\
\hline (Constante) & 6.23 & 0.70 & & $8.87^{*}$ \\
PLANIF. & 0.20 & 0.04 & .22 & $4.60^{*}$ \\
SUPERV. & 0.36 & 0.05 & .35 & $7.25^{*}$ \\
EVALUAC. & 0.28 & 0.04 & .30 & $6.23^{*}$ \\
\hline
\end{tabular}

$*_{p}<.001$

El análisis de regresión nos muestra que los tres procesos tienen valor predictivo a $p<$ .001 permitiendo explicar un $42 \%$ de la varianza de la composición escrita que se corresponde 
con un coeficiente de validez múltiple $\mathrm{R}=.64$. El hecho de que ninguno de los procesos sea eliminado del análisis de regresión por pasos, por posibles problemas de solapamiento o colinealidad (recuérdese que la correlación entre procesos era de .75) supone una evidencia adicional de que EVAPROMES mide tres procesos diferentes y que cada uno de ellos participa en la predicción de la producción escrita por separado.

La predicción del rendimiento académico es otra de las posibles aplicaciones de las pruebas metacognitivas. Como se observa en la Tabla 5 tanto EVAPROMES como ESCOLA muestran correlaciones estadísticamente significativas $(p<.001)$ con el rendimiento en diferentes asignaturas, con mayor fuerza en lengua española: si se toman ambas medidas como predictores de las calificaciones en lengua española vemos que obtenemos un coeficiente de validez predictiva de $\mathrm{R}=.43$ (ver Tabla 7). Observamos que ambas pruebas lejos de solaparse, se complementan y permiten hacer una mejor predicción del rendimiento académico.

Tabla 7. Modelo de regresión de las pruebas metacognitivas en las predicción de las calificaciones en lengua española.

\begin{tabular}{lcccc}
\hline & \multicolumn{2}{c}{ Coeficientes no } & \multicolumn{2}{c}{ Coeficientes } \\
estandarizados & tipificados & \\
& $B$ & Error típ. & $\beta$ & $t 1.43^{*}$ \\
\hline (Constante) & 50.36 & 4.41 & & $4.66^{*}$ \\
EVAPROMES & 0.49 & 0.10 & .29 & $3.73^{*}$ \\
ESCOLA & 0.21 & 0.06 & .23 & \\
\hline
\end{tabular}

$* p<.001$

\section{Fiabilidad}

EVAPROMES es un instrumento compuesto por ítems en una escala ordinal de tres puntos (puntuaciones 0,1 y 2), en estas situaciones se recomienda revisar la asimetría y apuntamiento (curtosis) de las distribuciones univariadas de los ítems, y en caso de que estas sean elevadas, estimar la matriz de correlaciones policóricas para los posteriores análisis y hacer la estimación de la fiabilidad mediante el coeficiente omega de McDonnald o GLB, puesto que el coeficiente alfa suele producir una subestimación de la fiabilidad en estos casos. Si bien, GLB solo debe utilizarse en muestras de tamaño moderado o grande $(n>500)$ para evitar sobre-estimaciones de la fiabilidad. 
En la muestra de Costa Rica, la fiabilidad de EVAPROMES se sitúa en un rango entre .80 y .86 dependiendo del procedimiento de estimación utilizado $(\alpha=.80, \omega=.84$ y $G L B=$ .86). En la muestra de España, la estimación de la fiabilidad según el procedimiento varía desde $\omega=.80, \alpha=.86$ hasta $G L B=.91$. En consecuencia, la fiabilidad de EVAPROMES se sitúa en ambas muestras en rangos similares con una estimación promedio de .85 , un valor adecuado para poder realizar comparaciones entre sujetos.

\section{Discusión y conclusiones}

EVAPROMES es un instrumento diseñado para evaluar metacompresión aplicada a la escritura, habiéndose validado las puntuaciones del instumento en dos poblaciones de habla hispana: costarricense y española. Los análisis de las puntuaciones obtenidas en ambas muestras sugieren que EVAPROMES es un instrumento con unas excelentes propiedades psicométricas tanto en su fiabilidad por consistencia interna como en su validez. La estructura del test acorde con la teoría en la que se basó la elaboración de los ítems, indica que en EVAPROMES se diferencian tres procesos: planificación, supervisión y evaluación, y que estos procesos se despliegan o desarrollan en función de tres variables (Persona, Tarea y Texto). Por lo tanto, si bien es posible obtener una medida de desempeño global, para una evaluación más precisa, cuando se desea realizar una intervención educativa, se recomienda obtener una medida de cada proceso y variable para obtener un perfil más ajustado de las habilidades metacognitivas del estudiante cuando escribe.

Un aspecto clave, y que generalmente se descuida en la elaboración de instrumentos, es la necesidad de obtener medidas invariantes, es decir, que antes de aplicar un instrumento para diferenciar entre grupos se tenga evidencia de que se está evaluando un mismo constructo. En este sentido, la presente investigación ha permitido mostrar que EVAPROMES mantiene el mismo constructo en dos poblaciones hispanohablantes bastante diferentes en sus características geográficas y culturales, lo que es un indicio sobre su posible generalización aunque esta conclusión deberá ser corroborada en ulteriores estudios con otras muestras de países hispanohablantes.

EVAPROMES ha mostrado ser útil para pronosticar la calidad de la producción escrita de los escolares, pero incluso más importante es el resultado que muestra cómo EVAPROMES y ESCOLA se relacionan con el rendimiento académico y el aprendizaje, confirmando 
la importancia de dominar las estrategias metacognitivas en el ámbito educativo (Coutinho, 2007; Dunning, Johnson, Ehrlinger y Kruger, 2003; Favieri, 2013, Kruger y Dunning, 1999). La autorregulación es un buen predictor del rendimiento académico en sus distintas facetas, el uso de estrategias de autorregulación como la planificación, la supervisión, la busqueda de objetivos y la perseverancia son esenciales para el logro académico en las diferentes tareas escolares (Álvarez-Valdivia, 2009; Corno, 1986; Martínez-Vicente y de la Fuente, 2004, Pintrich y De Groot, 1990; Zimmerman y Pons, 1986, 1988), así como la autoevaluación del propio alumno en todo su proceso de aprendizaje (Panadero y Alonso-Tapia, 2013). Los estudios correlacionales y de regresión lineal nos han permitido evaluar la relación entre el uso de estrategias metacognitivas aplicadas a la escritura, y otros constructos relacionados como la metacompresión en lectura (ESCOLA) o el clima de motivación en el aula (CMC-Q), la calidad de los textos escritos y el rendimiento académico. Se concluye que EVAPROMES se muestra como un instrumento con una alta capacidad para pronosticar la calidad de la producción escrita de los escolares, y junto con ESCOLA, como un buen predictor del rendimiento académico en dos áreas instrumentales básicas (lectura y escritura), recomendándose cuando sea posible combinar ambos instrumentos para diseñar didácticas más eficaces que reduzcan el fracaso escolar y, cuando sea preciso, hacer un mejor diagnóstico que permitirá realizar una intervención más eficaz.

Los educadores se han interesado por la escritura fundamentalmente como herramienta de desarrollo cognitivo, por este motivo, es importante conocer las estrategias metacognitivas que fomenten las habilidades de escritura: a) Hacer que los alumnos escriban con frecuencia; pocas actividades escolares producen más desarrollo cognitivo que hacer que los alumnos escriban a menudo y reciban retroalimentación sobre su escritura (Calkins, 1994), b) Crear un ambiente informal y de apoyo para la escritura, dicho ambiente promueve la escritura espontánea y creativa (Englert, Raphael, Anderson, Anthony y Stevens, 1991); c) Fortalecer las estrategias previas a la escritura como condición para organizar un plan de desarrollo (Bereiter y Scardamalia, 1987); d) Hacer hincapié en transformar el conocimiento en vez de narrarlo (Beal, 1996); e) Animar al alumno a desarrollar estrategias de revisión productiva, el diálogo con el grupo de iguales puede mejorar las estrategias de revisión (Englert et al., 1991); f) Emplear tecnología informática cuando sea preciso, particularmente el uso de los ordenadores puede facilitar la escritura de historias de calidad (Berninger, Vaughan, Abbott, Abbott, Rogan, Brooks, Reed y Graham, 1997). 
La habilidad de la escritura se desarrolla lentamente en la mayoría de los niños y adolescentes y solo alcanza la madurez en la edad adulta, y aun así, solo para algunos escritores (Bartlett, 2003). En la actualidad hay una gran preocupación por la cantidad de niños que no escriben o lo hacen mal en las escuelas. Un estudio estadounidense de ámbito nacional describía la escritura como la "gran ignorada" (National Commission on Writing, 2003). Aunque muchos alumnos de primaria y secundaria llegan a dominar los elementos básicos de la escritura, solo un reducido número, aproximadamente $25 \%$, escriben de manera competente. Hablar de escritura competente incluye "una buena organización dando respuestas convincentes y elaboradas a las tareas encomendadas y el uso de un lenguaje rico evocador y atractivo" (pp. 16-17). Para mejorar la escritura de los niños, la comisión recomienda que se duplique el tiempo dedicado a la escritura en la escuela; y que la escritura forme parte de todas las materias de estudio.

\section{Limitaciones e investigación futura}

Una de las limitaciones que observamos en EVAPROMES es el rango de edad que abarca. Sería necesario elaborar instrumentos que permitieran evaluar los procesos metacognitivos en escritura en toda la etapa de secundaria y bachillerato e incluso en estudiantes universitarios. Hemos observado que el alumnado preuniversitario y universitario español no aplica estrategias metacognitivas en tareas de escritura, por lo que consideramos interesante evaluar qué procesos y variables ponen en marcha cuando escriben. Si conseguimos un diagnóstico metacognitivo en este alumnado podremos implementar programas ad hoc para subsanar las deficiencias y así mejorar el rendimiento académico.

Aunque la prueba ha funcionado bien en dos países hispanohablantes, sería conveniente estudiar cómo se comporta el instrumento en otros países de lengua española. Las investigaciones futuras deberían incluir muestras de otros países con el fin de analizar la posible generalización de resultados a población hispanohablante con el objetivo de internacionalizar la prueba, y disponer de una herramienta útil para el diagnóstico y la intervención en lengua española. 


\section{Referencias}

Alonso-Tapia, J. y Fernández, B. (2008). Development and initial validation of the Classroom Motivational Climate Questionnaire (CMC-Q). Psicothema, 20(4), 883-889. Recuperado de http://www.redalyc.org/articulo.oa?id=72720456

Alvarez-Valdivia, I. M. (2009). Evaluar para contribuir a la autorregulación del aprendizaje. Electronic Journal of Research in Educational Psychology, 7(3), 10071030 .

American Educational Research Association, American Psychological Association, and National Council on Measurement in Education (AERA, APA, NCME, 2014). Standards for educational and psychological testing. Washington DC: American Psychological Association.

Bartlett, T. (2003). Why Johnny can't write, even though he went to Pricenton. Chronicle of Higher Education 49, A39. Recuperado de http://chronicle.com/free/v49/i17/17a03901.htm

Beal, C. R. (1996). The role of comprehension monitoring in children's revision. Educational Psychology Review, 8, 219-238

Bereiter, C. y Scardamalia, M. (1987). The psychology of written composition. Mahwah, New Jersey: Erlbaum.

Berninger, V. W., Vaughan, K. B., Abbott, R. D., Abbott, S. P. Rogan, L. W. Brooks, A., Reed, E. y Graham, S. (1997). Treatment of handwriting problems in beginning writers: Transfer handwriting to composition. Journal of Educational Psychology, 89, 652-666, doi: 10.1037/0022-0663.89.4.652

Calkins, L. M. (1994). The act of teaching writing. Portsmouth, NH: Heinemann.

Corno, L. (1986). The metacognitive control components of self-regulated learning. Contemporary Educational Psychology, 11, 333-346, doi: 10.1016/0361-476X(86)90029-9

Coutinho, S. A. (2007). The relationship between goals, metacognition, and academic success. Educate , 7 (1), 39-47.

Dunning, D., Johnson, K., Ehrlinger, J. y Kruger, J. (2003). Why people fail to recognize their own incompetence. Current Directions in Psychological Science, 12(3), 83-87, doi: $10.1111 / 1467-8721.01235$ 
Englert, R. S., Raphael, T., Anderson, L., Anthony, H. y Stevens, D. (1991). Making strategies and self-talk visible: Writing instruction in regular and special education classrooms. American Educational Research Journal, 28, 337-372.

Favieri, A. G. (2013). Inventario de estrategias meta-cognitivas generales (IEMG) e Inventario de estrategias meta-cognitivas en integrales (IEMI). Electronic Journal of Research in Educational Psychology, 11(31). 831-850, doi:10.14204/ejrep.3414095

Flavell, J. H. y Wellman, H. M. (1977). Metamemory. En R. V. Kail Jr. y J. W Hagen (Ed.). Perspectives on the development of memory and cognition (pp. 3-33). Hillsdale, N. J.: LEA.

Flórez, R., Mondragón, S.P., Pérez, C. y Torrado, M.C. (2003). Explorando la metacognición: evidencia en actividades de lectura y escritura en niños y niñas de 5 a 10 años de edad. Revista Colombiana de Psicología, 12, 85 - 98.

Flower, L. y Hayes, J. (1980). The dynamics of composing: making plans and jiggling constraints. En L. W. Gregg y E.R. Steinberg (Ed.). Cognitive processes in writing. (pp. 31-50). Hillsdale: LEA.

Galbraith, D. y Torrance, M. (1999). Conceptual processes in writing: from problema solving to text production. En M. Torrance y D. Galbraith (Ed.). Knowing what to write: conceptual processes in text production. Amsterdam: Amsterdam University Press.

Graham, S. y Harris, K.R. (1996). Self-regulation and strategy instruction for students who find writing and learning challenging. En C. M. Levy y S. Ransdell (Ed.). The science of writing: theories, methods, individual differences and applications. (pp. 347-360). Mahwah, N.J.: Earlbaum.

Graham, S. y Harris, K.R. (2000). The role of self-regulation and transcription skills in writing and writing development. Educational Psychologist, 1(35), 3-12, doi: 10.1207/S15326985EP3501_2.

Graves, D. (1996). Didáctica de la escritura. Madrid: Morata.

Hayes, J. (1996). A new framework for understanding cognition and affect in writing. En M.C. Levy y S. Ransdell (Ed.). The science of writing: theories, methods, individual differences and applications. (pp. 1-27). Mahwah, N.J.: Erlbaum.

Heller, M. y Thorogood, L. (1995). Hacia un proceso de lecto-escritura reflexivo y creativo. Caracas: Educativa.

Jiménez-Rodríguez, V., Puente-Ferreras, A., Alvarado-Izquierdo, J. M. y ArrebillagaDurante, L. A. (2009). Medición de estrategias metacognitivas mediante la Escala de 
Conciencia Lectora: ESCOLA. Electronic Journal of Research in Educational Psychology, 7(18), 779-804.

Karmiloff-Smith, A. (1995). Restricciones de la conciencia metalingüística. Infancia y Aprendizaje, 72, 33-50.

Kruger, J. y Dunning, D. (1999) Unskilled and unaware of it: How differences in recognizing one's own incompetence lead to inflated self-assessments. Journal of Personality and Social Psychology, 77(6), 1121-1134, doi:10.1037/0022-3514.77.6.1121

Lorenzo-Seva, U. y Ferrando, P.J. (2006). FACTOR: a computer program to fit the exploratory factor analysis model. Behavior Research Methods, 38(1), 88-91, doi: $10.3758 / \mathrm{BF} 03192753$

Martínez-Vicente, J.M. y de la Fuente, J. (2004). La Autorregulación del aprendizaje a través del programa Pro\&Regula. Electronic Journal of Research in Educational Psychology, 2(3), 145-156.

Mata, F. (1997). Dificultades en el aprendizaje de la expresión escrita. Málaga: Aljibe.

National Commission on Writing in America's Schools and Colleges (2003). The neglected " $R$ ": The need for a writing revolution. New York: The College Board.

Panadero, E. y Alonso-Tapia, J. (2013). Autoevaluación: connotaciones teóricas y prácticas. Cuándo ocurre, cómo se adquiere y qué hacer para potenciarla en nuestro alumnado. Electronic Journal of Research in Educational Psychology, 11(2), 551576, doi:10.14204/ejrep.3012200

Pintrich, P. R. y De Groot, E. V. (1990). Motivational and self-regulated learning components of classroom academic performance. Journal of educational psychology, 82(1), 33-40.

Puente-Ferreras, A., Jiménez-Rodríguez, V. y Alvarado-Izquierdo, J. M. (2009). ESCOLA. Escala de conciencia lectora. Madrid: EOS.

Scardamalia, M. y Bereiter, C. (1992). Dos modelos explicativos de los procesos de composición escrita. Infancia y Aprendizaje, 58, 43-64.

Shell, D. F., Murphy, C. C. y Bruning, R. H. (1989). Self-efficacy and outcome expectancy mechanisms in Reading and writing achievement. Journal of Educational Psychology, 81, 91-100, doi: 10.1037/0022-0663.81.1.91

Solé, I. y Teberosky, A. (2010). La enseñanza y el aprendizaje de la alfabetización. En C. Co11, J. Palacios, y A. Marchesi (Ed.). Desarrollo psicológico y educación. (pp. 462485). Barcelona: Alianza.

Tirapu-Ustárroz., J. y Muñoz-Céspedes, J.M. (2005). Memoria y funciones ejecutivas. Revista de Neurología, 41(8), 475-484. 
Ulate-Espinoza, M.A., Jiménez-Rodríguez, V., Alvarado-Izquierdo, J.M. y Puente-Ferreras. A. (2015). EVAPROMES. Evaluación de los procesos metacognitivos en escritura. Madrid: EOS.

Zimmerman, B. y Pons, M. (1986). Development of a structured interview for assessing student use of self-regulated learning strategies. American Educational Research Journal, 23, 614-628, doi: 10.3102/00028312023004614

Zimmerman, B. y Pons, M. (1988). Construct validation of a strategy model of student selfregulated learning. Journal of Educational Psychology, 80, 284-290, doi:10.1037/0022-0663.80.3.284 